\title{
Rise of pediatric robotic surgery in Italy: a multicenter observational retrospective study
}

Rossella Angotti ${ }^{{ }^{* \dagger}+}$, Alessandro Raffaele ${ }^{2 \dagger}$, Francesco Molinaro1,3, Giovanna Riccipetitoni ${ }^{2}$, Pierluigi Lelli Chiesa ${ }^{4}$, Gabriele Lisi ${ }^{4}$, Girolamo Mattioli ${ }^{5,6}$, Daniele Alberti ${ }^{7,8}$, Giovanni Boroni ${ }^{8}$, Francesca Mariscoli ${ }^{9}$, Ascanio Martino ${ }^{9}$, Gloria Pelizzo ${ }^{10}$, Michela Maffi" ${ }^{11}$, Mario Messina ${ }^{1,2}$ and Mario Lima ${ }^{11}$

\begin{abstract}
Background: The minimally invasive surgery (MIS) in term of robot-assisted surgery changed in a dramatic way the surgical approach either in adults or children. For many specialties (urology, gynecology, general surgery) robotic surgery rapidly became the gold standard for some procedures, while the experience in pediatric population is not wide for some reasons. The aim of this study is to retrospective analyze trends of application of robotic surgery in pediatric patients across the country, focusing on indications, limitations, development, and training acquired by national experience and in comparison to the literature.
\end{abstract}

Methods: We made a retrospective multicenter study on behalf of Italian Society of Pediatric Surgery. We performed a census among all pediatric surgery units in the country to enroll those performing robotic surgery on children between 2013 and 2019.

Results: We enrolled 7 pediatric surgery referral Centers (Ancona, Bologna, Brescia, Genova, Pavia, Pescara, Siena). A total of 303 patients were included in the study, 164 males (54\%) and 139 females (46\%). The most commonly performed interventions for each anatomic area were respectively atypical pulmonary resection (38\%), pyeloplasty (49\%), and fundoplication (30\%).

Conclusions: Since its first application in Italy, about 10 years ago, several considerations were made about application and feasibility of robotics in children.

Keywords: Robotic, Surgery, Child

\section{Background}

The minimally invasive surgery (MIS) era changed in a dramatic way the surgical approach both in adults and children [1]. The availability of small instruments and better magnification with a camera view allowed to perform all kinds of surgery obtaining better outcomes. In the last decade the introduction of robot-assisted surgery represents the further step in the evolution of MIS.

\footnotetext{
*Correspondence: rossellaangotti@me.com

${ }^{\dagger}$ Rossella Angotti and Alessandro Raffaele are equally contributed as co-first author.

${ }^{1}$ Division of Pediatric Surgery, Department of Women and Children, Le Scotte Hospital, Viale Bracci 16, 53100 Siena, Italy

Full list of author information is available at the end of the article
}

Surgical Robot as Da Vinci ${ }^{\circledR}$ offers three-dimensional (3D) visualization, a significantly improved instrumentation dexterity coupled with motion scaling, allowing surgeons to easily perform complex reconstructive procedures [2]. For some specialties, such as urology, gynecology and colorectal surgery, robotic surgery rapidly became the gold standard for some procedures in adults [3]. The experience in pediatric population is not wide for many reasons. The need to work in small and constrained cavities, the lack of instruments developed for children, the ratio between trocar and small body dimension, and the cost of the system represent the most common limits for application in children $[4,5]$. Since its first application in Italy about 10 years ago, several considerations 
were made about application and feasibility of robotics in children. The aim of our study is to retrospectively analyze trends of application of robotic surgery in pediatric patients across the country, focusing on indications, limitations, development and training acquired by national experience and in comparison to the literature.

\section{Methods}

We made a retrospective multicenter study on behalf of Italian Society of Pediatric Surgery.

We performed a census among all pediatric surgery units in the country to enroll those performing robotic surgery on children between 2013 and 2019. All the cases were treated with the da Vinci Surgical System ${ }^{\circledR}$ (Intuitive Surgical, Inc., Sunnyvale, CA, USA). From each center data collection included demographic patients' data (age, sex, diagnosis), pre-operative (nature of the procedure, operative time, conversion) and postoperative data (complications, length of stay). All data were

Table 1 Demographic data of the patients

\begin{tabular}{ll}
\hline Patients & 303 \\
Males/females & $164 / 139$ \\
Mean age at intervention & 144 months $(8-216 \mathrm{~m})$ \\
Mean weight at intervention & $38 \mathrm{~kg}(7-120 \mathrm{~kg})$ \\
Operative time & $158 \mathrm{~min}(40-450 \mathrm{~min})$ \\
Hospitalization & 7 days $(5-13$ days $)$ \\
Complications & $55(18 \%)$ \\
Conversions & $11(3 \%)$
\end{tabular}

identified. We divided surgical interventions into three groups, according to the anatomical district: thoracic, abdominal and genitourinary surgery.

\section{Statistics}

We used GraphPad Prism6 (GraphPad Software Inc., San Diego, CA, USA) for statistical analysis. Data were presented as mean $\pm \mathrm{SD}$, comparisons between groups performed using Student's $t$ test for unpaired data. A $p$ value $<0.05$ was considered significant.

\section{Results}

We enrolled 7 pediatric surgery referral Centers (Ancona, Bologna, Brescia, Genova, Pavia, Pescara, Siena). A total of 303 patients were included in the study, 164 males (54\%) and 139 females (46\%). All demographic data are summarized in Table 1. According to anatomic area the number of surgical interventions was 13 thoracic surgeries (6\%), 106 abdominal (34\%) and 184 genitourinary $(60 \%)$ as shown in Fig. 1. The most commonly performed interventions for each anatomical area were respectively atypical pulmonary resection (38\%), pyeloplasty (49\%), and fundoplication (30\%). The total number for each anatomic area is shown in Fig. 2a-c. Through the period of the study, we observed an overall increasing trend in the application of robotic surgery in children, with average one procedure a month for each center. In the last year of the study there was a decrease in trend due to the unavailability of the Robot among 2 centers, as shown in Fig. 3a. Mean operative time was $158 \mathrm{~min}$ with

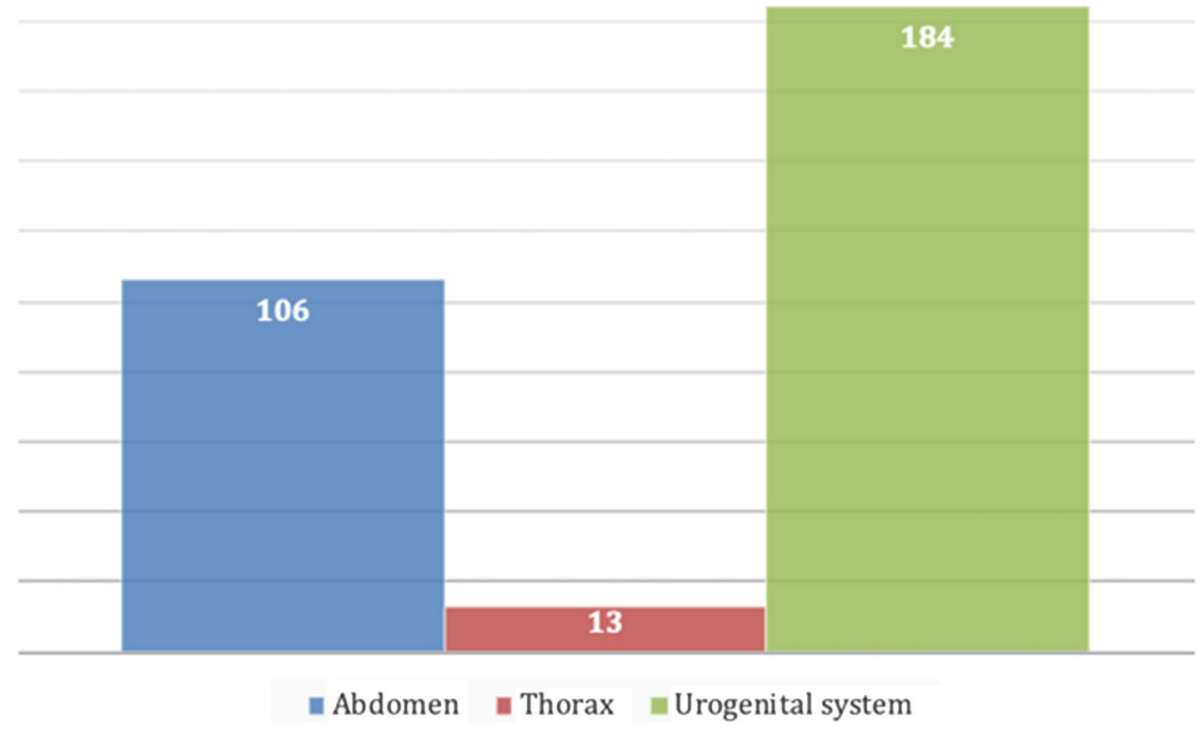

Fig. 1 Number of intervention for each anatomic district 

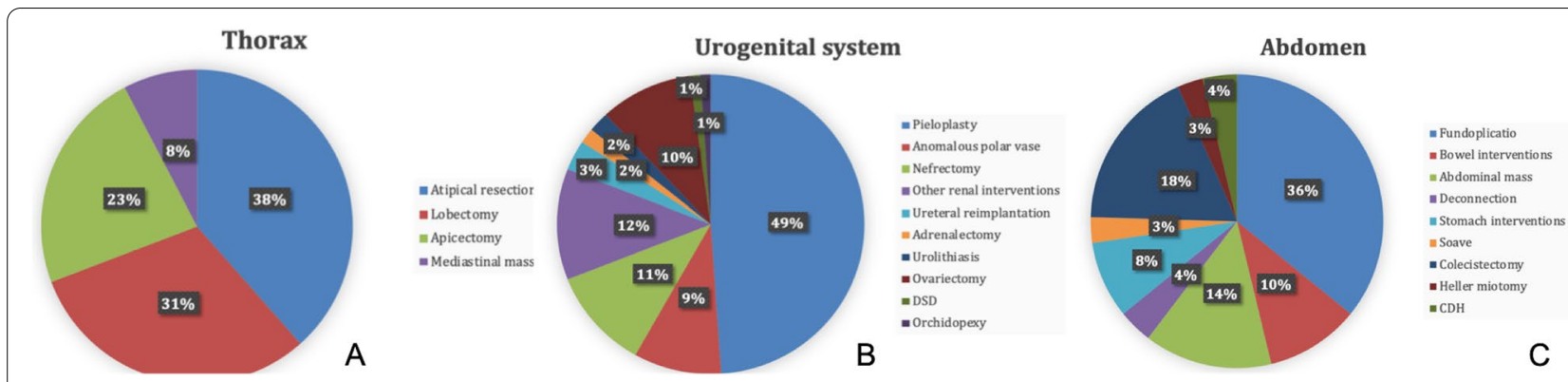

Fig. 2 a Main interventions performed about the thorax. b Main interventions performed about the genitourinary system. c Main interventions performed about the abdomen

no difference according to anatomic district (149 for thoracic surgery, 162 min for abdominal surgery and 148 for genitourinary surgery) (Fig. 3b).

Conversion rate was $3 \%$ : three patients for thoracic surgery, three patients for abdominal surgery and five patients for genitourinary surgery (Fig. 3c). Majority of complications occurred during thoracic surgery (61\%), followed by abdominal surgery $(16 \%)$ and genitourinary surgery (15\%) (Fig. 3d). There was no significant difference in terms of operative time, complications, and conversions between the three groups.

\section{Discussion}

Robot-assisted surgery, at the beginning, was conceived as a military tool for remote surgical care of the injured soldier, and later, in the 1990s, was introduced in the clinical practice [6]. Despite the fact that robotic surgery has reached high levels of expertise in adults with some procedures done as a gold standard yet the size and variety of available robotic instrumentation represent a limit for pediatric patients and the overall size of the robotic system can restrict the surgical indications [7]. The first report describing the use of robotic surgical systems for abdominal procedures in children published was published by Heller and colleagues in 2002 [8]. They reported a series of 11 children who underwent Thal or Nissen fundoplication for treatment of gastroesophageal reflux disease using a Da Vinci system. Mean patient age was 12 years and no complications were reported. From that time the applicability of robotic surgery in pediatric fields has made important progress and the indications for the intervention have been extended to other pathologies and to patients of lower age and weight. A retrospective study in 2019 [9] demonstrated that weight cannot be considered an absolute limit for robotic surgery. The improvement of instruments permits to perform complex surgical procedures in low-weight children without additional difficulties. Also, other studies [10,11] reported case series that demonstrated the safety and feasibility of robot in pediatric surgery. Our results confirm this process of growth in Italy: there is an evident and progressive increase in the number of interventions every year. This finding not only certified the rise of pediatric surgical indications, but also an improvement in the learning curve. A systematic review in 2013 showed an increase, through the years, both in case volumes of robotic surgical procedures in children and in the published literature on this subject. The Authors describe fundoplication, pyeloplasty and pulmonary resection as main procedures performed with robot [12]. These data are in agreement with our results that confirm a shared consent in considering robot-assisted surgery a gold standard for the treatment of gastroesophageal reflux and for ureteropelvic junction obstruction.

Several studies demonstrated no significant differences in outcomes and complications between laparoscopy and robot in the approach of these diseases [13-15]. In our case series, we observed a high prevalence of cholecystectomy and nephrectomy. There is a wide consensus in considering robotic unappropriated for these interventions, since both can be safely performed laparoscopically with a low risk of complications, lower cost, and same cosmetic result [16-21].

We could explain this application of robotic in Italy according to the availability of device. In many centers, pediatric surgeons share the robotic system with general surgeons and urologists, and this made possible to lower costs, widen surgical indications and increase the learning curve for robotic surgery. Thoracic surgery presents a different situation, with a few number of interventions performed both in our series and literature. In pediatric population majority of thoracic diseases are congenital (CPAM, lobar emphysema, lung sequestration) and they need an intervention in the first months of life, which is impossible due to lack of suitable instrument size, until now, the use of robot according to unavailability of small size instruments. Even in toddlers, the thoracic cavity is small and trocar positioning with triangulation and are 


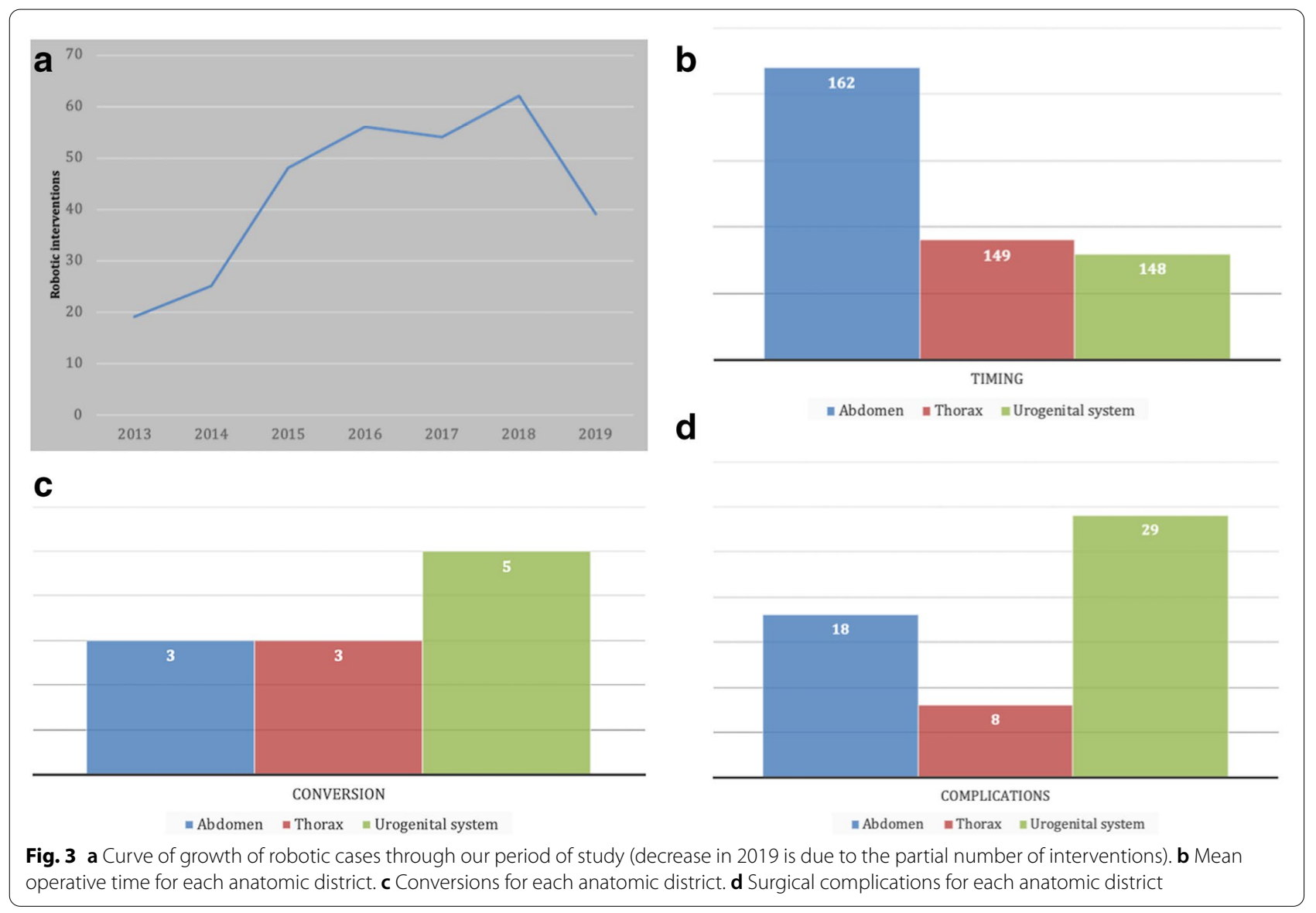

difficult. There is also a need for single lung ventilation that makes it a challenge also for the anesthesiologists [5]. In the Italian experience, the pulmonary partial resection for the treatment of pleural blebs is the most common indication for thoracic robotic surgery. This is a common disease in teenagers and young adults, who present dimensions of thorax more suitable for robotic approach.

\section{Conclusions}

The results of this work give the reason to believe that in Italy, as in the rest of Europe and in the USA, pediatric robotic surgery is a field in development which presents a progressive growth. Pyeloplasty and fundoplication are, to date, the most frequent surgeries performed in children, where outcomes are recognized as at least equivalent to the open or laparoscopic procedures. The robotic pyeloplasty is the most commonly practiced procedure with the least operative complications in our series. Many other procedures have been reported, and still under evaluation with more data expected in the near future. A further increase of learning curve and improvements of robotic surgery can be the next step to do to widen the application of robot-assisted surgery. Further prospective studies comparing the robotic surgery with the conventional techniques to justify why should we dwell on such expensive approach.

\section{Abbreviations}

MIS: Minimally invasive surgery; CPAM: Congenital pulmonary airway malformations.

\section{Acknowledgements \\ Not applicable.}

\section{Authors' contributions}

All authors equally contributed to collect analyzed and interpreted data. Rossella Angotti and Alessandro Raffaele were major contributors in writing the manuscript. Prof. Mario Lima was a supervisor. All authors read and approved the final manuscript.

\section{Funding}

No funding for this article. No personal financial interests.

\section{Availability of data and materials}

All data generated or analyzed during this study are included in this published article. The original datasets are available by each institution's dataset.

\section{Declarations}

Ethics approval and consent to participate Not applicable. 


\section{Consent for publication}

Not applicable.

\section{Competing interests}

All authors declare that they have no competing interests.

\section{Author details}

${ }^{1}$ Division of Pediatric Surgery, Department of Women and Children, Le Scotte Hospital, Viale Bracci 16, 53100 Siena, Italy. ${ }^{2}$ Department of Pediatric Surgery, Fondazione IRCCS Policlinico San Matteo, Viale Camillo Golgi 19, 27100 Pavia, Italy. ${ }^{3}$ Division of Pediatric Surgery, Department of Medical, Surgical and Neurological Sciences, University of Siena, Siena, Italy. ${ }^{4}$ Department of Pediatric Surgery, Pescara Hospital, Via Renato Paolini 47, 65124 Pescara, Italy. ${ }^{5}$ DINOGMI, University of Genoa, Genoa, Italy. ${ }^{6}$ Paediatric Surgery Unit, Giannina Gaslini Children's Hospital, Institute for Scientific Research, Via Gerolamo, Gaslini, 5, 16147 Genova, Italia. ${ }^{7}$ Department of Paediatric Surgery, "Spedali Civili" Children's Hospital, 25123, Via del Medolo, 2, 25123 Brescia, BS, Italy. ${ }^{8}$ Department of Clinical and Experimental Sciences, University of Brescia, 25123 Brescia, Italy. ${ }^{9}$ Pediatric Surgery Unit, Salesi Children's Hospital, Università Politecnica delle Marche, Via Filippo Corridoni, 11, 60123 Ancona, Italy. ${ }^{10}$ Ospedale dei Bambini V. Buzzi, Via Castelvetro, 32, 20154 Milano, Italy. ${ }^{11}$ Department of Pediatric Surgery, Sant'Orsola Hospital, University of Bologna, Via Giuseppe Massarenti, 9, 40138 Bologna, Italy.

\section{Received: 1 August 2021 Accepted: 2 November 2021}

Published online: 03 February 2022

\section{References}

1. Andolfi C, Kumar R, Boysen WR, Gundeti MS. Current status of robotic surgery in pediatric urology. J Laparoendosc Adv Surg Tech A. 2019;29(2):159-66.

2. Suematsu Y, del Nido PJ. Robotic pediatric cardiac surgery: present and future perspectives. Am J Surg. 2004;188(4A Suppl):98S-103S. https://doi. org/10.1016/j.amjsurg.2004.08.003.

3. Moore LJ, Wilson MR, Waine E, Masters RSW, McGrath JS, Vine SJ. Robotic technology results in faster and more robust surgical skill acquisition than traditional laparoscopy. J Robot Surg. 2015;9:67-73.

4. Spinoit AF, Nguyen H, Subramaniam R. Role of robotics in children: a brave new world! Eur Urol Focus. 2017;3:172-80.

5. Molinaro F, Krasniqi P, Scolletta S, et al. Considerations regarding pain management and anesthesiological aspects in pediatric patients undergoing minimally invasive surgery: robotic vs laparoscopic-thoracoscopic approach. J J Robot Surg. 2020;14(3):423-30.

6. Chandra V, Dutta S, Albanese CT. Surgical robotics and image guided therapy in pediatric surgery: Emerging and converging minimal access technologies. Seminars in Pediatric Surgery. 2006;15:267-75.

7. Hollands CM, Dixey LN. Applications of robotic surgery in pediatric patients. Surg Laparosc Endosc Percutan Tech. 2002;12:71-6.

8. Heller K, Gutt C, Schaeff B, Beyer PA, Markus B. Use of the robot system Da Vinci for laparoscopic repair of gastro-oesophageal reflux in children. Eur J Pediatr Surg. 2002;12:239-42.
9. Molinaro F, Angotti R, Bindi E, et al. Low weight child: can it be considered a limit of robotic surgery? Experience of Two Centers. J Laparoendosc Adv Surg Tech A. 2019;29(5):698-702.

10. Friedmacher $F$, Till H. Robotic-assisted procedures in pediatric surgery: a critical appraisal of the current best evidence in comparison to conventional minimally invasive surgery. J Laparoendosc Adv Surg Tech A. 2015;25:936-43.

11. Lima M, Thomas E, Di Salvo N, Gargano T, Ruggeri G. Paediatric surgery in the robotic era: early experience and comparative analysis. La Pediatria Medica e Chirurgica. 2019;41:204.

12. Cundy TP, Shetty K, Clark J, Pin CT, Sriskandarajah K, Gattas NE, et al. The first decade of robotic surgery in children. J Pediatr Surg. 2013;48:858-65.

13. Hubens G, Coveliers H, Balliu L, Ruppert M, Vaneerdeweg W. A performance study comparing manual and robotically assisted laparoscopic surgery using the da Vinci system. Surg Endosc. 2003;17:1595-9.

14. Cundy TP, Harling L, Hughes-Hallett A, et al. Meta-analysis of robotassisted vs conventional laparoscopic and open pyeloplasty in children. BJU Int. 2014;114:582-94.

15. Mattioli G, Wong MCY, Angotti R, et al. Total oesophago-gastric dissociation in neurologically impaired children: Laparoscopic vs robotic approach. Int J Med Robot. 2020;16(1):e2048.

16. Camps J. The use of robotics in pediatric surgery: my initial experience. Pediatr Surg Int. 2011;27:991-6.

17. Bansal D, Cost NG, Bean CM, et al. Comparison of pediatric roboticassisted laparoscopic nephroureterectomy and laparoendoscopic singlesite nephroureterectomy. Urology. 2014;83:438-42.

18. Goruppi I, Avolio L, Romano P, Raffaele A, Pelizzo G. Robotic-assisted surgery for excision of an enlarged prostatic utricle. Int J Surg Case Rep. 2015;10:94-6.

19. Pelizzo G, Nakib G, Romano P, et al. Five millimetre-instruments in paediatric robotic surgery: advantages and shortcomings. Minim Invasive Ther Allied Technol. 2015;24(3):148-53.

20. Nakib G, Calcaterra V, Goruppi I, et al. Robotic-assisted surgery approach in a biliary rhabdomyosarcoma misdiagnosed as choledochal cyst. Rare Tumors. 2014;6(1):5173

21. Mattioli G, Molinaro F, Paraboschi I, et al. Robotic-assisted minimally invasive total esophagogastric dissociation for children with severe neurodisability. J Laparoendosc Adv Surg Tech A. 2017;27(5):550-5.

22. Pelizzo G, Nakib G, Goruppi I, et al. Pediatric robotic pyeloplasty in patients weighing less than $10 \mathrm{~kg}$ initial experience. Surg Laparosc Endosc Percutan Tech. 2014;24(1):e29-31.

23. Davies B. A review of robotics in surgery. Proc Inst Mech Eng. 2000;214:129-40. https://doi.org/10.1243/0954411001535309.

24. Sim HG, Yip SK, Cheng CW. Equipment and technology in surgical robotics. World J Urol. 2006;24:128-5.

\section{Publisher's Note}

Springer Nature remains neutral with regard to jurisdictional claims in published maps and institutional affiliations.

\section{Submit your manuscript to a SpringerOpen ${ }^{\circ}$ journal and benefit from:}

- Convenient online submission

- Rigorous peer review

- Open access: articles freely available online

- High visibility within the field

- Retaining the copyright to your article

Submit your next manuscript at springeropen.com 